\title{
Efficient Haptic Rendering of Regolith
}

\author{
Aaron Pereira $^{1,2}$ and Annika Schmidt ${ }^{1,3}$
}

\begin{abstract}
Driven by the need for physically accurate and haptically convincing models of lunar and planetary regolith for model-mediated teleoperation in space, we present an approach to modelling regolith in an efficient yet realistic way. Model parameters are derived from physical characteristics of the regolith, to render regoliths with different density profiles, cohesion, internal friction and in different gravitational fields.

Users could distinguish between changes in parametersspecifically friction and gravity field-and also gave qualitative feedback relevant to modelling regolith. We discuss the challenges in haptically rendering soils and the next steps.
\end{abstract}

\section{INTRODUCTION}

Haptic rendering of non-rigid media is an understudied, yet valuable field. Use cases include teleoperated undersea cable laying, immersive gaming experiences, or modelmediated teleoperation with interaction with sands, soils or gravels. Our motivation is exploration and in-situ resource utilisation (ISRU) on heavenly bodies. An efficient, haptically realistic and physically faithful model of regolith has many uses here, for example in astronaut training or mission simulation in virtual reality, or in teleoperation. Modelmediated teleoperation [1] is useful in the space context where communication is unreliable or delayed: the operator interacts with a local model of the remote environment without delay, the remote robot follows operator commands on a high level, and the local model is updated from remote sensing. To minimise updates from the remote side due to discrepancies between real and modelled behaviour, the local model must approximate the behaviour of the real regolith accurately. Physically realistic behaviour also improves the usefulness of virtual reality training or simulation.

While rendering of rigid objects with viscoelastic models, and soft materials with e.g. the Hunt-Crossley model [2], [3] has been studied extensively, haptic interaction with fluids and regolith is less developed. Fluid and regolith dynamics are calculation-intensive, limiting use in haptics where high update rates are key. Moving a tool through regolith, in contrast to fluid, not only deforms, but also compacts the regolith, changing its material properties. Recent work shows that even when rendering only characteristic properties such as viscosity [4], or inertia [5], [6], users can perceive media to be convincingly fluid. This begs the question: could a simplified model suffice for regolith interaction?

\footnotetext{
${ }^{1}$ Institute of Robotics and Mechatronics, German Aerospace Center, Oberpfaffenhofen, Germany aaron.pereira@dlr.de

${ }^{2}$ Human-Robot Interaction Lab, European Space Agency, Noordwijk, Netherlands

${ }^{3}$ Dept. of Informatics, Sensor Based Robotic Systems and Intelligent Assistance Systems, TU Munich, Germany annika.schmidtedlr.de
}

The mechanics of soils and powders has been investigated for e.g. industrial processes, civil engineering or geomechanics [7], [8]. In the space domain, the use case is predicting the properties of regolith on heavenly bodies for excavation [9] or sampling activities in low-gravity conditions [10].

The relationships between the physical properties of the soil or powder constituents (e.g particle size distribution, surface roughness, shape, chemical composition and material density) and engineering properties (cohesion force per area, internal friction angle, angle of repose, bulk density, etc.) have been much studied theoretically, and estimations made for lunar regolith from empirical studies [11], [12].

From Discrete Element Modelling (DEM), insights into the behaviour of regolith under conditions difficult to test in a lab (e.g. different gravity or atmosphere, or particle profiles) can be derived. For example, particle shape is crucial to cohesion [13], and lunar regolith is highly irregularly-shaped. Approaches to modelling the non-sphericity of soils and powders involve introducing wrenches at the contact points, or modelling with non-spherical particles [14].

Similar to Smoothed-Particle Hydrodynamics (SPH) for fluid rendering [15], we represent the regolith by individual particles. Our method must be accurate enough to simulate large-scale behaviour of regoliths, provide a convincing haptic feel, and still be fast enough for a haptic loop of $1 \mathrm{kHz}$. To this end we first represent the regolith by macroparticles (or pseudoparticles), see e.g. [16]. In our model these are identical, compressible spheres which reflect the material properties of the regolith in their dynamics and their interactions with each other. Having reduced the number of bodies to model, we further reduce calculations in interparticle interactions (which are $O\left(n^{2}\right)$ in number of particles $n$ ), by simplifying the dynamics while upholding realistic regolith behaviour.

In the following section, we make static analyses to show how cohesion, internal friction and bulk density of the regolith correspond to the parameters of our simplified model. We perform a short user validation in Sec. III to gauge how well users can differentiate varying material properties and their perception of the modelled regolith. We conclude in Sec. IV, discussing in Sec. V limitations and next steps.

\section{SoIL Engineering Properties}

There are three main parameters of the regolith which are relevant in our modelling:

1) the bulk density $\rho$, which is related to the void ratio (ratio of the voids to solid regolith) and the density of the rock of which the regolith consists; 
2) cohesion $C$, i.e. the resistance of particles to being pulled apart or moving past each other when there are no normal compressive forces. This can be due to moisture, or in dry material, due to electrostatic and van der Waals forces between fine-grained particles [7]

3) friction coefficient $\mu$, related to the particle shapes, and also the roughness of the material. Commonly this is given as friction angle $\phi$, where $\mu=\tan (\phi)$

We next show how these engineering parameters of soil relate to our model parameters: $R$, the radius of a macroparticle; $\rho_{0}$ and $\rho_{\max }$, the surface and maximum bulk densities; and $\gamma$ and $\hat{\mu}$, relating to the cohesion and friction.

\section{A. Bulk Density}

Measurements of lunar regolith show that the bulk density $\rho$ increases rapidly with depth in the first few centimetres. Based on measurements from Apollo mission samples, an empirical model can be fitted [11]:

$$
\rho=\rho_{0}+\kappa \ln (100 z+1)
$$

where $z \geq 0$ is the depth in metres, the surface bulk density is $\rho_{0}=1270 \mathrm{kgm}^{-3}$ and the constant $\kappa=0.121$. This model is valid as far as the measurements are made, i.e. $0.6 \mathrm{~m}$. Densely packed regolith in deeper layers may require drilling, and given the abundance of regolith over the lunar surface, it is likely that only the top few centimetres would be excavated for ISRU applications [17]. A model valid for the first $0.6 \mathrm{~m}$ is therefore adequate.

During simulations, it was observed that the spherical macroparticles settled into a close-packing, see Fig. 1. Each layer bears the weight of the $n$ layers above it. In this analysis, it is assumed that the layers stretch infinitely to the horizon, so the horizontal forces on each particle are net zero; we also assume no horizontal stresses, so no penetration between spheres on the same layer. In the following analysis we have:

$R \quad$ macroparticle radius (we choose $R=7 \mathrm{~mm}$ )

$m \quad$ mass of one macroparticle,

$n$ number of layers above the current,

$d \quad$ depth of the layer,

$\rho(d) \quad$ bulk density as a function of depth $d$,

$g \quad$ acceleration due to gravity,

$b(g, m, n)$ vertical distance between layer $n$ and $n-1$,

$a(g, m, n)$ distance between a macroparticle in layer $n$ and

its neighbour in layer $n-1$.

See Fig. 1. The vertical distance between the centres of spheres in adjacent layers is $b$, where $b$ is a function of $g, m$, and $n$ (we drop the dependencies of $b$ in the following for legibility). Hence the volume occupied by a single macroparticle in layer $n$ (including voids) is:

$$
V(n)=2 \sqrt{3} R^{2} \frac{(b(n)+b(n+1))}{2}
$$

At the surface $(n=0)$ there is no weight from above, so:

$$
\begin{aligned}
& b(0)=\frac{2 \sqrt{2}}{\sqrt{3}} R \\
& m=4 \sqrt{2} \rho_{0} R^{3}
\end{aligned}
$$
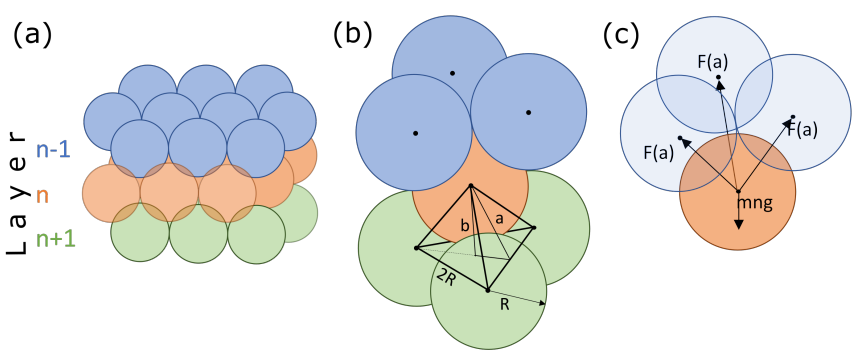

Fig. 1. Vertical compressive forces on a macroparticle (a) the macroparticles settle into a close packing (b) vertical distance $b$ and distance $a$ between centres of closest macroparticles in neighbouring layers (c) free body diagram of forces on a macroparticle in layer $n$.

We determine how $b$ decreases with increasing $n$ and thus increasing load of the layers above. Consider the free body diagram in Fig. 1(c). In the lattice structure each macroparticle bears the weight of three macroparticles from the layer above, but is supported by three macroparticles from the layer below, hence, the total force borne by a macroparticle in layer $n$ is $m n g$ (the top layer is layer 0 ). This is split over the three macroparticles it supports, hence the vertical component of each of the three forces $F(a)$ in the free-body diagram, is $\frac{n m g}{3}$. The distance $a$ between the centre of a sphere and one of its nearest neighbours in the layer above is:

$$
a=\sqrt{b^{2}+\frac{4 R^{2}}{3}}
$$

The vertical component of the reaction force $F(a)$ in the macroparticle balances the weight from the particle above:

$$
\frac{b}{a} \cdot F(a)=\frac{n m g}{3}
$$

A linear viscoelastic model, i.e. $F(a)=k(2 R-a)$, does not yield correct behaviour in the first few centimetres, see Fig. 2. We therefore consider a model where force is inversely proportional to penetration distance:

$$
\|F(a)\|= \begin{cases}0 & a \geq 2 R \\ \frac{\gamma}{a-a_{\min }}-\frac{\gamma}{2 R-a_{\min }} & a_{t h r} \leq a<2 R \\ k a_{t h r}+F\left(a_{t h r}\right)-k a & a<a_{t h r}\end{cases}
$$

Where $a_{t h r}=a_{\min }+\sqrt{\frac{\gamma}{k}}, d=\frac{\gamma}{2 R-a_{\min }}, \gamma$ is a constant to be found and the theoretical boundary $a_{\min }$ is so chosen that the density of the layer at this boundary is as high or higher than the regolith at minimum porosity/void ratio. From [11], void ratio is as low as 0.7 (meaning porosity of $41 \%$ ) with a specific gravity of 3100 , leading to a maximum density of around $\rho_{\max } \approx 1820 \mathrm{kgm}^{-3}$ or above. Since in each layer the macroparticles are always $2 R$ apart, density is inversely proportional to $b$, so with some trigonometry using (5):

$$
a_{\min }=\frac{2 R}{\sqrt{3}} \sqrt{2 \cdot\left(\frac{\rho_{0}}{\rho_{\max }}\right)^{2}+1}
$$

The spring constant $k$ is a limiting stiffness after which $F(a)$ behaves linearly, i.e. like a spring-without it, forces would tend asymptotically to infinity as $a \rightarrow a_{\min }$. Higher 


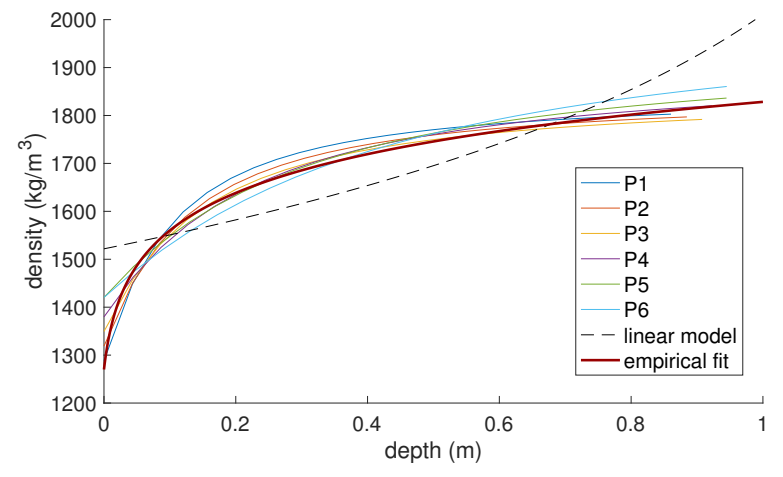

Fig. 2. The models with the parameters from Tab. II, the linear viscoelastic force model with $k=100 \mathrm{Nm}^{-1}$, and the logarithmic model from [11] .

stiffness means higher damping to keep the system stable [18], see Sec. II-D. We found $k=100 N / m$ to be adequate, but this depends on hardware and haptic loop frequency. In the following analysis, we assume arbitrarily high $k$ for generality.

Letting $h=\frac{\gamma}{2 R-a_{\min }}$, substituting the inverse-proportional formula from (7) into (6), multiplying by $a\left(a-a_{\min }\right)$, and rearranging:

$$
b\left(\gamma+h a_{\min }\right)-a^{2} \frac{n m g}{3}=a\left(b h-a_{\min } \frac{n m g}{3}\right)
$$

Substituting (5) and squaring both sides, we obtain a quartic polynomial expression for $b$ :

$$
\begin{aligned}
\left(b\left(\gamma+h a_{\min }\right)-\right. & \left.\left(b^{2}+\frac{4 R^{2}}{3}\right) \frac{n m g}{3}\right)^{2}= \\
& \left(b^{2}+\frac{4 R^{2}}{3}\right)\left(b h-a_{\min } \frac{n m g}{3}\right)^{2}
\end{aligned}
$$

The bulk density $\rho$ is inversely proportional to the value of $b$, and from (3) and (2) we get:

$$
\rho(n)=\frac{4 \sqrt{2} R \rho_{0}}{\sqrt{3}(b(n)+b(n+1))}
$$

Further, the depth $d$ as a function of $n$ is:

$$
d(n)=\frac{b(0)}{2}+\sum_{i=1}^{i=n} b(i) .
$$

While an analytical solution to the quartic in (10) does exist, the discrete integration in (12) inhibits an explicit expression for $\rho$ as a function of $d$. However, both can be evaluated for values of $n$ and compared with the literature. In Fig. 2 we see the density profiles for the sets of parameters in Table II which are used in our user validation. In Tab. I the densities from these parameter sets are compared with empirical measurements in [11].

\section{B. Cohesion and Internal Friction}

Lunar regolith is fine grained, with a mean particle size of 60-90 microns [19], thus cohesion forces are relevant. Also, whereas air and moisture form a separating layer between particles in terrestrial soils, in a vacuum particles can get
TABLE I

COMPARISON OF DENSITY VALUES FROM [11] VS OURS WITH VARIOUS PARAMETER SETS $\left(\mathrm{kgm}^{-3}\right)$

\begin{tabular}{|l|c|c|c|c|c|c|c|}
\hline Depth & Empirical [11] & P1 & P2 & P3 & P4 & P5 & P6 \\
\hline $0-15 \mathrm{~cm}$ & $1500 \pm 50$ & 1501 & 1498 & 1501 & 1501 & 1516 & 1503 \\
$0-30 \mathrm{~cm}$ & $1580 \pm 50$ & 1593 & 1584 & 1579 & 1575 & 1582 & 1566 \\
$30-60 \mathrm{~cm}$ & $1740 \pm 50$ & 1759 & 1747 & 1737 & 1744 & 1745 & 1741 \\
$0-60 \mathrm{~cm}$ & $1660 \pm 50$ & 1676 & 1666 & 1658 & 1660 & 1664 & 1654 \\
\hline
\end{tabular}

closer and cohesive surface forces (which decrease rapidly with distance) and friction are larger. This theory is backed up by experiments [20], [21] and DEM predicting an increase in shear strength of $12-15 \%$ on the moon compared to earth [9]. The Coulomb relation relates shear forces of friction $\tau_{f r}$ to normal stress $\sigma_{n}$, the coefficient of internal friction $\mu$ and the force of cohesion per unit effective area $C$ [7]:

$$
\tau_{f r}=\mu \sigma_{n}+C .
$$

The friction angle $\phi$ is frequently given in literature; $\mu$ relates to the friction angle by $\mu=\tan (\phi)$.

1) Simplified Model: Inter-particle friction forces depend on the relative velocities of particles. This would also be $O\left(n^{2}\right)$, hence calculation-intensive. Instead, we base the modelled friction and cohesion forces on a particle on the absolute velocity. We propose the following model to account for both friction and cohesion forces on a particle:

$$
\left\|F_{f r, c}\right\| \begin{cases}\leq \hat{\mu} \Sigma\left\|F_{\text {contact }}\right\| & \|\dot{\boldsymbol{x}}\|=0 \\ =\lambda \hat{\mu} \Sigma\left\|F_{\text {contact }}\right\| & \|\dot{\boldsymbol{x}}\|>0\end{cases}
$$

Where $\Sigma\left\|F_{\text {contact }}\right\|$ sums the magnitudes of contact forces between macroparticles determined according to (7), and $\dot{x}$ is the particle velocity. The top case is a threshold value necessary to set a stationary particle in motion; the bottom case is the resistive force when the particles are in motion. This is lower than 1: in [13], normal stress decreased after yielding, suggesting that the friction in regolith in flow may be lower than when static. In practice, setting $\lambda=1$ led to slow creep of the particles; a value of $\lambda=0.8$ was adequate to avoid this.

2) Internal Friction: To find a value for $\hat{\mu}$, we analyse (13), and relate it to values reported in the literature. In [12], values of $30^{\circ}-50^{\circ}(0.6 \leq \mu \leq 1.2)$ and $0.1 k P a \leq C \leq$ $1 k P a$ are reported for the Apollo 15 mission. Other studies have yet higher values of cohesion [22].

Consider macroparticles in a close packing, as in Fig. 3(a), isotropically compacted to a density of $\rho_{1}=1967 \mathrm{kgm}^{-3}$. Hence the distance between adjacent macroparticles is:

$$
a_{1}=2 R \sqrt[3]{\frac{\rho_{0}}{\rho_{1}}}
$$

An increase in compressive stress $d \sigma$ results in an increase in compressive force $d F=d \sigma A$ on a single macroparticle, where $A=\frac{\sqrt{3}}{2} a_{1}^{2}$ is the area on which it acts. The three macroparticles contacting the macroparticle from above, and the three from below, share the force, i.e. the vertical component from each of these is $\frac{d F}{3}$, and by trigonometry, 

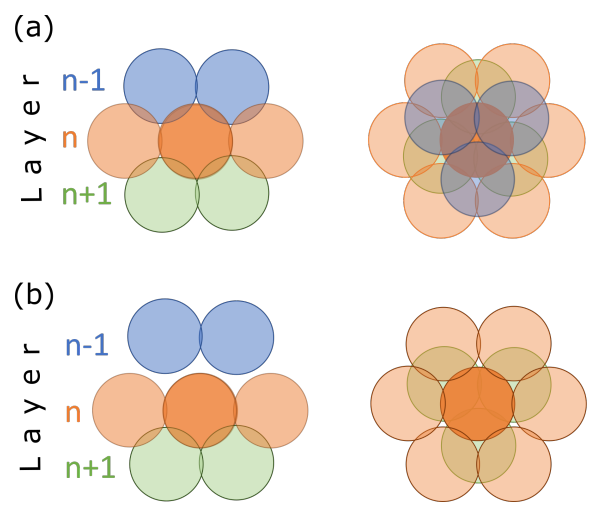

(c)

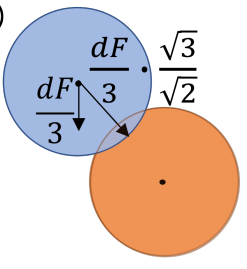

Fig. 3. (a) a macroparticle has 12 neighbours, of which 3 in the layer above and 3 in the layer below (b) detaching layers $n-1$ and $n$ to remove compressive force at the boundary, a macroparticle in layer $n$ intersects 9 neighbours, (c) increase in force of penetration and its vertical component.

the force of penetration for each of these 6 macroparticles is $\frac{d F}{3} \sqrt{\frac{3}{2}}$ (Fig. 3(c)). Summing the additional forces from these macroparticles leads to an increase of $\sqrt{6} \hat{\mu} d F=\sqrt{6} \hat{\mu} d \sigma_{n} A$ in the force $F_{f r, c}$ needed to set the particle in motion when it is stationary, given in (14). Assuming $C$ in (13) to be independent of normal stress, the increase in friction force over the effective area of the macroparticle, $d \tau_{f r} A$, is then:

$$
d \tau_{f r} A=\mu d \sigma_{n} A=\sqrt{6} \hat{\mu} d \sigma_{n} A,
$$

leading to an expression for coefficient of internal friction:

$$
\mu=\sqrt{6} \hat{\mu}
$$

3) Cohesion: Cohesion can be calculated by separating two layers so that there is no penetration and thus no compressive force on the bottom layer, see Fig 3(b). The resistance to motion of a macroparticle in this layer is $9 \hat{\mu} F\left(a_{1}\right)=9 \hat{\mu} \gamma\left(\frac{1}{a_{1}-a_{\min }}-\frac{1}{2 R-a_{\min }}\right)$, with $a_{\min }$ as defined in (8). The effective area of each macroparticle in the layer is $A=\frac{\sqrt{3}}{2} a_{1}^{2}$. Thus the force of cohesion on the macroparticle:

$$
F_{C}=9 \hat{\mu} \gamma\left(\frac{1}{a_{1}-a_{\min }}-\frac{1}{2 R-a_{\min }}\right)
$$

and per unit area:

$$
C=\frac{6 \sqrt{3} \hat{\mu} \gamma}{a_{1}^{2}}\left(\frac{1}{a_{1}-a_{\min }}-\frac{1}{2 R-a_{\min }}\right) .
$$

With values of $3 \times 10^{-5} \leq \gamma \leq 1.2 \times 10^{-4}$ and values of $0.25 \leq \hat{\mu} \leq 0.5$ (corresponding to friction angles of $30-50^{\circ}$ as in [12]), cohesion works out typically between $0.5-2 k P a$, which are values for regolith similar to those in [22].

\section{Cohesion Between Noncontacting Macroparticles}

The simulated cohesion and friction forces in the previous section act only when macroparticles intersect. But what about electrostatic and van der Waals forces causing particles to clump together? This behaviour is difficult to determine empirically or theoretically: such forces act in the range of
$10^{-8} m$ [13], however, we observe even on the earth how fine dust clumps and coheres to itself.

We model it as an attractive force at the particle edge. For fast calculation, we simply modify (7) to:

$$
\|F(a)\|= \begin{cases}0 & a \geq 2 R_{a t t r} \\ \frac{\gamma}{a-a_{\min }}-\frac{\gamma}{2 R-a_{\min }} & a_{t h r} \leq a<2 R_{a t t r} \\ k a_{t h r}+F\left(a_{t h r}\right)-k a & a<a_{t h r}\end{cases}
$$

From empirical tests, large values (e.g. $R_{a t t r}=1.25 R$ ) cause behaviour similar to surface tension, whereas $R_{a t t r}=$ $R$ exhibits behaviour similar to plasticine or wet sand. We found $R_{a t t r}=1.1 R$ led to convincing regolith behaviour.

\section{Damping}

In a discretised virtual spring, the interaction forces between macroparticles also need to be damped in order to maintain stability. However, inter-particle calculations should be minimised for efficiency. Similarly to friction (Sec. II-B), not relative speed but absolute speed is used in damping. Damping is thus based on the stability criterion of [18]:

$$
F_{d a m p}=-\frac{p k \Delta t}{2} \dot{\boldsymbol{x}}
$$

where $p$ is the number of macroparticles contacting the macroparticle, $k$ is the limiting stiffness in (19), and $\Delta t$ is the timestep of the haptic loop.

\section{E. Interaction with tool}

The interaction with the tool must be kept crisp yet stable; we also consider cohesion to the tool. We opted for a viscoelastic model for each macroparticle:

$$
\|F(z, \dot{z})\|=\left\{\begin{array}{ll}
0 & z \leq-0.002 R \\
k_{\text {tool }} z+c_{\text {tool }} \dot{z} & z>-0.002 R
\end{array},\right.
$$

where $z$ is the penetration of the macroparticle and tool, and $\dot{z}$ is their relative velocity; the force direction opposes penetration. The forces and torques on the tool from all macroparticles are then summed. The tool shape was simply a rectangular "shovel", aligned with the gripper of the Sigma.7 device. To increase efficiency, macroparticles were first collision-checked with a sphere which encloses the tool, then collision checked with the tool. The parameters of the viscoelastic model were: $k_{t o o l}=500 \mathrm{~N} / \mathrm{m}, c_{t o o l}=$ 1.4. $\frac{\Delta t k_{\text {tool }}}{2}$, where $\Delta t$ is the timestep of the haptic loop.

At high values of $\hat{\mu}$ and $m$, vibration was sometimes observed when the tool was contacting several compacted particles on both of its largest faces; filtering forces and torques on the tool with a low-pass filter of cutoff frequency $30 \mathrm{~Hz}$, removed this while not affecting crispness of contact.

Cohesive forces were applied similarly to Sec. II-C, i.e. with a small viscoelastic attractive component outside penetration, since lunar dust readily adheres to surfaces [23]. A value of $0.002 R$ gave realistic-appearing behaviour - in lunar gravity some macroparticles would adhere to the tool but could be removed by shaking. 


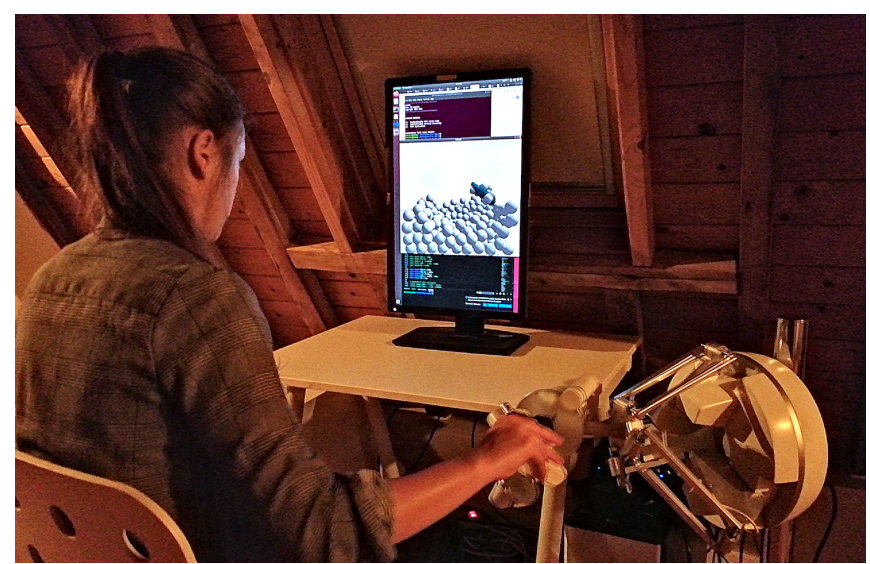

Fig. 4. Setup with test subject

\section{USER VALIDATION OF HAPTICS}

A user validation was carried out in form of experiments to determine whether the haptic perception was realistic and how well the friction and weight of the regolith could be perceived. For this purpose, the algorithm was implemented using the CHAI3D framework [24] on a 4-core Intel i7-3820 at $3.6 \mathrm{GHz}$ with 16GB RAM and an NVIDIA Quadro K600 graphics card. The haptic device used was a Force Dimension sigma.7. The setup is shown in Fig. 4.

There were 256 macroparticles in the simulation arranged into a hexagonal close-packing three layers deep and bounded by a floor and by vertical walls enclosing a space $126 \mathrm{~mm} \times 126 \mathrm{~mm}$. The sigma.7 controlled the movement of a virtual spatula tool in the simulation, of size $50 \mathrm{~mm} \times 40 \mathrm{~mm} \times 5 \mathrm{~mm}$, which had no weight but reflected forces and torques acting on the tool to the user. It was also not constrained by the virtual walls, allowing the user to move the tool under the regolith.

\section{A. Methodology}

Eight users (4 female, 3 male, 1 genderqueer) interacted with the virtual regolith. 2 were left-handed, 5 right-handed, 1 ambidextrous; interaction was performed with the right hand since the device fit a right hand. Users' perception capabilities for two characteristic behaviours were investigated. In one trial, gravity constant $g$ was changed, leading to different perception of the regolith weight. Surface gravity values were chosen of Ceres $\left(0.270 \mathrm{~ms}^{-2}\right)$, Pluto $\left(0.625 \mathrm{~ms}^{-2}\right)$, Europa $\left(1.314 m s^{-2}\right)$, Mars $\left(3.721 \mathrm{~ms}^{-2}\right)$ and Earth $\left(9.807 \mathrm{~ms}^{-2}\right)$, with the reference of the Moon $\left(1.625 \mathrm{~ms}^{-2}\right)$ and parameter set P4 in Table II. In a second trial, internal friction $\mu$ was changed, leading to a more "sticky" or "smooth" feeling during the interaction. In order to keep cohesion the same, since parameter $\hat{\mu}$ also affects cohesion, the parameter sets in Table II were chosen, keeping constant cohesion of $C=$ $0.97 \mathrm{kPa}$ while varying friction, and fitting the bulk density profile as in Sec. II-B.2. The reference was parameter set P3, values of $0.6 \leq \mu \leq 1.2$ corresponded to friction angles in the range of $30-50^{\circ}$ as in [12].
TABLE II

PARAMETER SET

\begin{tabular}{|l|c|c|c|c|c|c|}
\hline $\begin{array}{c}\text { Parameter } \\
\text { set }\end{array}$ & $\begin{array}{c}\text { Desired } \\
\mu\end{array}$ & $\hat{\mu}$ & $\gamma(\mathrm{Nm})$ & $\begin{array}{c}\rho_{0} \\
\left(\mathrm{kgm}^{-3}\right)\end{array}$ & $\begin{array}{c}\rho_{\max } \\
\left(\mathrm{kgm}^{-3}\right)\end{array}$ & $\begin{array}{c}g \\
\left(\mathrm{~ms}^{-2}\right)\end{array}$ \\
\hline P1 & 1.21 & 0.494 & $3 \times 10^{-5}$ & 1270 & 1824 & 1.625 \\
P2 & 1.00 & 0.409 & $3.5 \times 10^{-5}$ & 1300 & 1824 & 1.625 \\
P3 & 0.835 & 0.342 & $4 \times 10^{-5}$ & 1330 & 1824 & 1.625 \\
P4 & 0.735 & 0.300 & $6 \times 10^{-5}$ & 1360 & 1880 & 1.625 \\
P5 & 0.648 & 0.265 & $8 \times 10^{-5}$ & 1400 & 1920 & 1.625 \\
P6 & 0.600 & 0.245 & $1.2 \times 10^{-4}$ & 1400 & 2000 & 1.625 \\
\hline
\end{tabular}

In each trial, a forced-choice procedure was applied, in which the user was first presented with a reference condition, then with a test stimulus set either above or below the value of the reference. The user had to indicate if the tested condition was "heavier" (varying $g$ ) or "more resistant to motion" (varying $\hat{\mu}$ ) than the reference. Within each trial, each condition was encountered thrice and the order was pseudo-random. Four participants started with the trial varying gravity, while four started with the trial varying friction. In each trial the percentage of times that a test stimulus was perceived "heavier"/"more resistant" than the reference stimulus was plotted as a function over the varying parameter. For values much lower than the reference, the percentage tends toward zero; for much higher values it will tend to $100 \%$, indicating that the participants can easily distinguish the two stimuli. Between extrema, a smooth transition from $0-100 \%$ is expected, which can be fitted with a psychometric function of the form

$$
f(x)=50 \%+50 \% \cdot \operatorname{erf}\left(\frac{\log (x / p)}{\sqrt{2} \log (w+1)}\right),
$$

where $\operatorname{erf}$ is the error function, $p$ the reference stimulus and $w$ the Weber fraction as a free parameter. This fraction-a common psychometric measure-indicates the relative change of a given stimulus the user needs to perceive a difference.

In Fig. 5 the obtained psychometric curves for the users' perception capabilities for a varying $\mu(5 a)$ and $g(5 b)$ are shown. In the friction experiment the characteristic S-Shape could be seen and over all participants a Weber fraction of 0.29 can be found. This suggests that an absolute increase of 0.24 in $\mu$ is needed for the users to perceive a change in regolith behaviour. The characteristic S-Shape for gravity was found when plotted on a logarithmic x-scale. As the $g$ values were also chosen with this in mind, based on [25], this was expected. The Weber fraction for gravity was 3.31: a threefold increase or decrease in gravity would be necessary to perceive a change.

\section{B. Discussion}

The user validation verified that the developed algorithm could display simplified, yet seemingly realistic, regolith behaviour. One user reported that the interaction was reminiscent of interacting with dust. Another user reported that it did not feel like interacting with distinct balls but rather with one mass, which felt $d r y$. This confirms the intention of the 
(a)

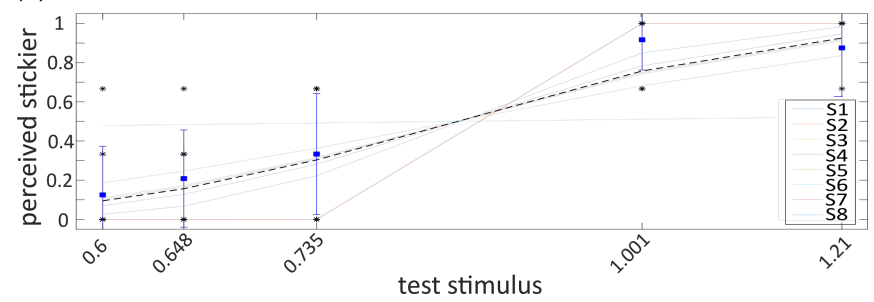

(b)

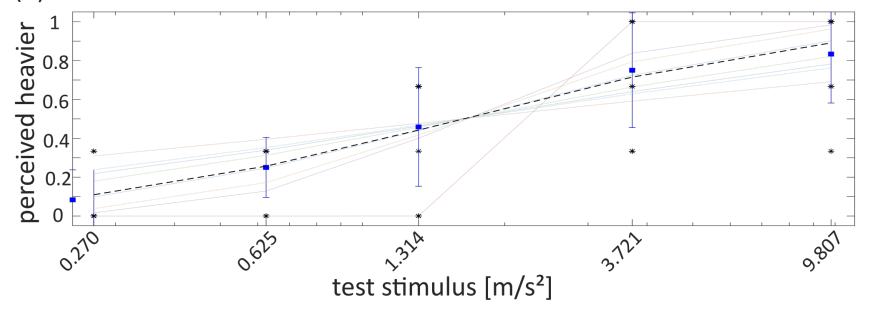

Fig. 5. Psychometric curves for subjects S1-S5, for the experiment with (a) friction $\mu$ and (b) gravity $g$

developers to give the impression of a continuous material rather than discrete particles.

Our user validation can only give limited insight into the perception of friction and gravity by users of our modelfor more insight, more subjects and more interaction steps would be necessary. Nevertheless, it was clear that subjects were able to distinguish these properties. Furthermore, the Weber fraction of 0.29 for friction is close to that found for fluids with high viscosity in real experiments [26] as well as in a haptic setup [4].

The higher Weber fraction for gravity perception is due to the larger step sizes but could also be because humans are not used to perceiving different gravity values. On Earth, weight and mass are perceived the same, but the inertial properties of the regolith were constant regardless of the force of gravity. That is to say, the same user force applied perpendicular to gravity would result in the same movement of regolith. We did not observe significant differences in the user's peak force in different gravitational conditions. One user remarked that, while we explore properties such as friction actively, by moving our hand or a tool through a medium and registering the resistive forces, gravity is perceived on the own body or on objects the user is holding. Since the tool had no weight in the simulation, users tended to use a scooping motion to gauge the weight of the regolith. To gauge friction, they used a stirring action or compacted the regolith against a wall.

Because the user could move the tool underneath and outside the virtual walls containing the regolith, it was deemed necessary for the users to also see the visual representation (see video attachment). Visual cues influence haptic perception [27], and users were able to see that particles were more sticking together in the friction experiment and the changing behaviour of falling particles in different surface gravity was visible. Not all users used the visual cues to a large extent, and when asked about this after the experiment, some did not realise that these were relevant or even suspected that they might be deliberately out of sync with the haptics to test haptic vs. visual perception. The influence of the visuals is nevertheless suspected to have supported the haptic perception, though not investigated here.

\section{CONCLUSION}

This paper presents a preliminary approach to an efficient yet sufficiently accurate model to render regolith for virtual reality and model-mediated teleoperation. Engineering properties such as bulk density, cohesion and internal friction coefficient are related to the simplified dynamics of interacting macroparticles. With this model, a variety of properties of regolith can be simulated. Users are able to distinguish between different gravity conditions and different internal friction. Although this research is still in an early stage, it is an important first step towards model-mediated teleoperation with regoliths and soils, which will be a crucial technology in future (robotic-aided) exploration of other worlds.

\section{LimitaTiONS AND OUTLOOK}

Some limitations can be addressed in future research. Computational complexity is still $O\left(n^{2}\right)$, because interparticle interactions are considered. However, keeping these as efficient as possible reduces computation. Methods whereby a macroparticle is only collision-checked with a limited subset of neighbours could reduce complexity to $O(n)$.

To improve realistic haptic perception, while maintaining computational efficiency, a hybrid model could be considered, with finer-grained particles interacting with the tool with coarser macroparticles or even continuum mechanics used for the rest of the simulation, such as in [28]. Additionally, a more detailed user study could determine characteristics most relevant for realistic perception and study users' perception capabilities in detail.

Friction and damping acting on the absolute velocity of particles means that clumps of particles falling or sliding are artificially slowed. A falling clump of particles has no resistance to its motion (in a vacuum) and a sliding clump has friction only from the sliding surface. Different friction models could be investigated and compared to DEM simulations. Compared to terrestrial soil mechanics, it is difficult to validate regolith models in space conditions. However, model-mediated teleoperation experiments can validate the physical properties of the model (i.e. not just haptic perception) on regolith simulant on Earth at least.

Besides particle-based methods, voxel-based approaches have been trialled [29], whose advantage is complexity of $O(n)$, where $n$ is the number of voxels. However, conservation of mass must be guaranteed, which is implicit in particlebased methods. The challenge of rendering haptics in voxelbased methods is an open problem, but an interesting one.

\section{ACKNOWLEDGEMENT}

Many thanks to the subjects in the user validation, and to the Human Robot Interaction Lab at ESA, for allowing us to monopolise their sigma.7 device. 


\section{REFERENCES}

[1] P. Mitra and G. Niemeyer, "Model-mediated telemanipulation," Int. J. Robotics Research, vol. 27, no. 2, pp. 253-262, 2008.

[2] K. H. Hunt and F. R. E. Crossley, "Coefficient of restitution interpreted as damping in vibroimpact," J. Appl. Mech, vol. 42, no. 2, pp. 440445, 1975.

[3] C. Liu, J. Guo, and P. Poignet, "Nonlinear model-mediated teleoperation for surgical applications under time variant communication delay," IFAC-PapersOnLine, vol. 51, no. 22, pp. 493 - 499, 2018, 12th IFAC Symposium on Robot Control SYROCO 2018.

[4] A. Schmidt, A. Pereira, T. Baker, B. Pleintinger, T. Hulin, Z. Chen, D. A. Abbink, and N. Y. Lii, "Enabling interaction with virtual fluids and mixed media using a high dexterity hand exoskeleton," in Proc. IEEE Int. Conf. on Systems, Man and Cybernetics, 2020.

[5] Z. Wang and Y. Wang, "Haptic interaction with fluid based on smooth particles and finite elements," in Int. Conf. on Comput. Science and Its Applications. Springer, 2014, pp. 808-823.

[6] M. Vines, J. Mora, and W.-S. Lee, "Real-time haptic display of fluids," in Proc. 2nd Canadian Conf. on Computer Science and Software Engineering, 2009, pp. 149-153.

[7] R. L. Brown and J. C. Richards, Principles of Powder Mechanics. Pergamon PreMss, 1970.

[8] C. O'Sullivan, Particulate discrete element modelling: a geomechanics perspective. Spon Press, 2011.

[9] C. Chang and P.-Y. Hicher, "Model for granular materials with surface energy forces," Journal of Aerospace Engineering, American Society of Civil Engineers, vol. 2, no. 9, pp. 43-52, 2009.

[10] J. Brisset, J. Colwell, A. Dove, S. Abukhalil, C. Cox, and N. Mohammed, "Regolith behavior under asteroid-level gravity conditions: low-velocity impact experiments," Progress in Earth and Planetary Science, vol. 5, no. 73, 2018.

[11] W. N. Houston, J. K. Mitchell, and W. D. Carrier III, "Lunar soil density and porosity," in Proc. 5th Lunar Sci. Conf., vol. 3, 1972, pp. 2361-2364.

[12] J. K. Mitchell, W. N. Houston, R. F. Scott, N. C. Costes, W. D. Carrier, III, and L. G. Bromwell, "Mechanical properties of lunar soil: Density, porosity, cohesion, and angle of internal friction," in Proc. 3rd Lunar Sci. Conf., vol. 3, 1972, pp. 3235-3253.

[13] M. Jiang, Z. Shen, and C. Thornton, "Microscopic contact model of lunar regolith for high efficiency discrete element analyses," Computers and Geotechnics, vol. 54, pp. $104-$ 116, 2013.

[14] J. Kozicki, J. Tejchman, and Z. Mróz, "Effect of grain roughness on 13 strength, volume changes, elastic and dissipated energies during quasistatic homogeneous triaxial compression using dem," Granular Matter, vol. 14, pp. 457-468, 2012.

[15] G. Cirio, M. Marchal, S. Hillaire, and A. Lecuyer, "Six degrees-offreedom haptic interaction with fluids," IEEE Trans. Visualization and Computer Graphics, vol. 17, no. 11, pp. 1714-1727, 2011.

[16] R. Hoffmann, "DEM simulations of toner particles with an $\mathrm{O}(\mathrm{N} \log$ N) hierarchical tree code algorithm," Granular Matter, vol. 8, no. 151, 2006.

[17] G. Just, K. Smith, K. Joy, and M. Roy, "Parametric review of existing regolith excavation techniques for lunar in situ resource utilisation (isru) and recommendations for future excavation experiments," Planetary and Space Science, vol. 180, no. 104746, 2020.

[18] J. E. Colgate and J. M. Brown, "Factors affecting the z-width of a haptic display," in Proceedings of the 1994 IEEE International Conference on Robotics and Automation, vol. 4, 1994, pp. 3205-3210.

[19] D. S. McKay, G. H. Heiken, R. M. Taylor, U. S. Clanton, D. A. Morrison, and G. H. Ladle, "Apollo 14 soils: Size distribution and particle types," in Proc. 3rd Lunar Sci. Conf., vol. 1, 1972, pp. 983994.

[20] O. R. Walton, C. P. De Moor, and K. S. Gill, "Effects of gravity on cohesive behavior of fine powders: implications for processing lunar regolith," Granular Matter, vol. 9, pp. 353-363, 2007.

[21] L. G. Bromwell, "Model for granular materials with surface energy forces," MIT, Tech. Rep. R66-18, 1966.

[22] C. W. D., O. G. R., and M. W., "Physical properties of the lunar surface," in Lunar sourcebook: a user's guide to the moon, H. G. H., V. D. T., and F. B. M., Eds. Cambridge University Press, 1991, pp. 475-567.

[23] J. Gaier, "The effects of lunar dust on EVA systems during the Apollo missions," NASA, Tech. Rep. NASA/TM-2005-213610, 2005.
[24] F. Conti, F. Barbagli, R. Balaniuk, M. Halg, C. Lu, D. Morris, L. Sentis, J. Warren, O. Khatib, and K. Salisbury, "The chai libraries," in Proceedings of Eurohaptics 2003, Dublin, Ireland, 2003, pp. 496500.

[25] R. D. Portugal and B. F. Svaiter, "Weber-fechner law and the optimality of the logarithmic scale," Minds \& Machines, vol. 21, pp. 73-81, 2011.

[26] W. M. Bergmann Tiest, A. C. Vrijling, and A. M. Kappers, "Haptic discrimination and matching of viscosity," IEEE Trans. Haptics, vol. 6, no. 1, pp. 24-34, 2013.

[27] G. Buckingham, J. S. Cant, and M. A. Goodale, "Living in a material world: how visual cues to material properties affect the way that we lift objects and perceive their weight," J. Neurophysiology, vol. 102, no. 6, pp. 3111-3118, 2009.

[28] M. Servin, T. Berglund, and S. Nystedt, "A multiscale model of terrain dynamics for real-time earthmoving simulation," 2020.

[29] A. D. Geiger, "A voxel-based approach to the real-time simulation of sands and soils," 2015, Master's Thesis. 